\title{
Assessment of the $1 \%$ of Patients with Consistent $<15 \%$ Reduction in Low-Density Lipoprotein Cholesterol: Pooled Analysis of 10 Phase 3 ODYSSEY Alirocumab Trials
}

\author{
Harold E. Bays ${ }^{1} \cdot$ Robert S. Rosenson ${ }^{2} \cdot$ Marie T. Baccara-Dinet ${ }^{3} \cdot$ Michael J. Louie $^{4} \cdot$ Desmond Thompson $^{4}$. \\ G. Kees Hoving ${ }^{5}$
}

Published online: 7 April 2018

(C) The Author(s) 2018

\begin{abstract}
Purpose Clinical trials of statins and other lipid-lowering therapies (LLTs) often report large inter-individual variations in their effects on low-density lipoprotein cholesterol (LDL-C). We evaluated apparent hyporesponsiveness to the proprotein convertase subtilisin/kexin type 9 inhibitor alirocumab (defined as $<15 \%$ LDL-C reduction from baseline at all timepoints) using data from 10 Phase 3 trials (3120 hypercholesterolemic patients).

Methods This report assessed the LDL-C percent reduction from baseline at weeks 4-104 (depending on study), and alirocumab serum levels and antidrug antibodies, in patients with apparent hyporesponsiveness.

Results Among the 3120 patients evaluated, $98.9 \%$ responded to alirocumab, and 33 (1.1\%) had < 15\% LDL C reduction at all measured timepoints. Pharmacokinetics data indicated that 13/33 apparent hyporesponders had not received alirocumab; no pharmacokinetics data were available for $14 / 33$, and $6 / 33$ had detectable alirocumab. For the six patients with confirmed alirocumab receipt, the degree of adherence to pre-study concurrent LLTs could not be determined after study start; one of these patients had persistent antidrug antibodies.

Conclusions Apparent hyporesponsiveness to alirocumab appeared to be due to lack of receipt of alirocumab determined by serum alirocumab levels, possible lack of adherence to concurrent LLTs, a theoretical and rare possibility of biological non-responsiveness due to persistent antidrug antibodies, or other causes, as yet unidentified.
\end{abstract}

Keywords Low density lipoprotein cholesterol $\cdot$ Cholesterol-lowering drugs $\cdot$ PCSK9 $\cdot$ Antibodies

Electronic supplementary material The online version of this article (https://doi.org/10.1007/s10557-018-6784-z) contains supplementary material, which is available to authorized users.

Harold E. Bays

hbaysmd@outlook.com

1 Departments of Epidemiology \& Medicine, Louisville Metabolic and Atherosclerosis Research Center (L-MARC), 3288 Illinois Avenue, Louisville, KY 40213, USA

2 Cardiometabolics Unit, Mount Sinai Heart, Icahn School of Medicine at Mount Sinai, New York, NY, USA

3 Clinical Development, R\&D, Sanofi, Montpellier, France

4 Regeneron Pharmaceuticals, Inc., Tarrytown, NY, USA

5 Department of Vascular Medicine - Internal Medicine Academic Medical Center, University of Amsterdam,

Amsterdam, The Netherlands

\section{Introduction}

Clinical trials of lipid-lowering therapies (LLTs), including statins, often report large inter-individual variations in their effects on low-density lipoprotein cholesterol (LDL-C), including apparent lack of response in some patients. Potential causes of hyporesponsiveness may be lack of receipt of active study drug, changes in concurrent LLTs, inaccurate or unrepresentative baseline lipid levels, or biological non-responsiveness [1]. Race/ethnicity, smoking status, or age may also be factors [2].

Alirocumab is a fully human monoclonal antibody to proprotein convertase subtilisin/kexin type 9 (PCSK9). In high cardiovascular risk patients (including those with heterozygous familial hypercholesterolemia $[\mathrm{HeFH}]$ ) from Phase 3 ODYSSEY studies, mean LDL-C change from baseline to Week 24 with alirocumab at $150 \mathrm{mg}$ every 2 weeks (Q2W) was -45.7 to $-61.0 \%$ versus +0.8 to $-6.6 \%$ with placebo, 
and at $75 \mathrm{mg}$ with possible increase to $150 \mathrm{mg} \mathrm{Q} 2 \mathrm{~W}(75 / 150)$ at week 12 was $-48 \%$ versus +9.1 to $-2.3 \%$ [3]. Alirocumab was generally well tolerated in the trials $[3,4]$. This analysis of 3120 alirocumab-treated patients with available data from 10 Phase 3 ODYSSEY studies examined apparent hyporesponsiveness to alirocumab, defined as $<15 \%$ LDL-C reduction from baseline at all study timepoints wherein blood samples were obtained.

\section{Methods}

This analysis evaluated patients with baseline LDL-C levels $\geq 70 \mathrm{mg} / \mathrm{dl}$ pooled from 10 ODYSSEY placeboor ezetimibe-controlled trials who were treated with alirocumab at $75 / 150$ or $150 \mathrm{mg}$ Q2W, with or without statin and/or other LLTs [5-13]. In the trials, a total of 3120 alirocumab-treated patients were included in the modified intent-to-treat population (all randomized patients with an LDL-C assessment on at least one post-baseline timepoint while on treatment). Trials were at least 24 weeks in duration, with four trials of 78 weeks; one trial was 104 weeks (Supplemental Fig. 1). All trial protocols were approved by the appropriate regulatory body, and all participants were provided written, informed consent.

The LDL-C percent reduction from baseline was examined at weeks 4, 8, 12, 16, 24, 36, 52, 64, 78 (or 76), 88, and 104, depending on the study. The $<15 \%$ cutoff for hyporesponsiveness was chosen based on the general minimum LDL-C reduction required for drug approval by the US Food and Drug Administration [14] and was used to define lack of response to PCSK9 inhibitors in previous analyses $[15,16]$.

Treatment adherence to planned injections of alirocumab and antidrug antibodies were examined in all hyporesponders, as defined above. Serum alirocumab levels $\left(\mathrm{PK}_{\mathrm{Ali}}\right)$ were available for a subset of these patients. Alirocumab intake was confirmed when $\mathrm{PK}_{\mathrm{Ali}}$ was $>10 \%$ of the expected alirocumab concentration on at least one timepoint.

\section{Laboratory Analyses}

Patient blood samples for lipid analysis were taken under fasting conditions and were assayed by central laboratories (Medpace Reference Laboratories, Cincinnati, OH, and Leuven, Belgium, except for the LONG TERM study [12], which used Covance Central Laboratories, Indianapolis, IN, and Geneva, Switzerland). Total cholesterol, triglycerides, and high-density lipoprotein cholesterol (HDL-C) levels in serum were determined using Centers for Disease Control and Prevention National Heart Lung Blood Institute Lipid Standardization Program assays. LDL-C was calculated using the Friedewald formula (total cholesterol minus HDL-C minus triglycerides/5); unless triglyceride levels were $>400 \mathrm{mg} /$
$\mathrm{dL}$, in which case it was determined by ultracentrifugation and precipitation (beta-quantification) by the central laboratory. Apolipoprotein B and lipoprotein(a) levels in serum were determined using immunonephelometry. Alirocumab and PCSK9 concentrations in serum were quantified with validated enzyme-linked immunosorbent assays (Regeneron Pharmaceuticals Inc., Tarrytown, NY, USA) [17]. Serum samples were tested for anti-drug antibodies to alirocumab using a validated immunoassay; samples positive for anti-drug antibodies were screened for neutralizing antibodies using a validated immunoassay (Regeneron Pharmaceuticals Inc., Tarrytown, NY, USA) [18].

\section{Results}

Alirocumab at 75/150 mg Q2W resulted in LDL-C reduction of $\geq 15 \%$ in $91 \%$ of the patients at week 12 (before dosage increase) and in $92 \%$ of the patients at week 24 (Supplemental Fig. 2A). Approximately $95 \%$ of the patients treated with alirocumab at $150 \mathrm{mg}$ Q2W observed an LDL-C reduction of $\geq 15 \%$ at weeks 12 and 24 (Supplemental Fig. 2B). Across all studies, alirocumab produced an LDL-C reduction of $<15 \%$ on at least one timepoint in $\sim 25 \%$ of patients (Supplemental Fig. 3); however, only 1.1\% $(n=33)$ had $<15 \%$ LDL-C reduction at all timepoints. Patient diary/caregiver reports indicated $\geq 80 \%$ adherence to planned alirocumab injections in all 33 patients; seven of these 33 patients were from studies with no pharmacokinetics analysis per protocol. Of the 26 patients where $\mathrm{PK}_{\mathrm{Ali}}$ data were available, 13 had undetectable $\mathrm{PK}_{\mathrm{Ali}}$, suggesting no intake of alirocumab. Two had $\mathrm{PK}_{\mathrm{Ali}}$ values missing, and five discontinued treatment early (Fig. 1).

The remaining six patients with $<15 \%$ LDL-C reduction had detectable $\mathrm{PK}_{\mathrm{Ali}}$ levels. All were White, with ages ranging 20-63 years; five had HeFH [15] (Table 1). Per protocol, all six patients were to have continued concurrent maximally tolerated statin after study entry; however, adherence to concurrent statins was not specifically monitored. This presented a potential confounder in that if high-intensity statin was discontinued after the study had started, then it would be expected to contribute to subsequent intra-individual LDL-C increases, potentially negating the apparent efficacy of alirocumab. Overall adherence to alirocumab was reported as $>80 \%$ in all six patients, but only two completed treatment up to the end of the trial. One patient who reported being treated with atorvastatin $80 \mathrm{mg}$ per day at study start had persistent antidrug antibodies but stopped treatment at week 36 due to poor adherence to protocol (Table 1). It is unknown whether the patient also had poor adherence to concurrent statin. Persistent antidrug antibodies were found in $<2 \%$ of alirocumab-treated patients in the 10 Phase 3 trials, which did not seem to affect efficacy in the broad patient population [18]. 
Fig. 1 Flow chart of LDL-C response to alirocumab treatment pooled from 10 Phase 3

ODYSSEY trials. ${ }^{a}$ Excluding one patient without post-baseline LDL-C data. ${ }^{\mathrm{b}} \mathrm{PK}$ Ali data available for only one or two timepoints (Weeks 0,4 , and/or 8 ) as patient discontinued treatment early. Due to early treatment discontinuation, no conclusions can be made based on the $\mathrm{PK}_{\mathrm{Ali}}$ findings in those patients. Pooled on-treatment data from 10 Phase 3 ODYSSEY trials: COMBO I, NCT01644175; LONG TERM, NCT01507831; HIGH FH, NCT01617655; FH I, NCT01623115; FH II,

NCT01709500; COMBO II,

NCT01644188; MONO,

NCT01644474;

ALTERNATIVE,

NCT01709513; OPTIONS I,

NCT01730040; and OPTIONS

II, NCT01730053. $L D L-C$, low-

density lipoprotein cholesterol;

$P K_{A l i}$, serum alirocumab levels

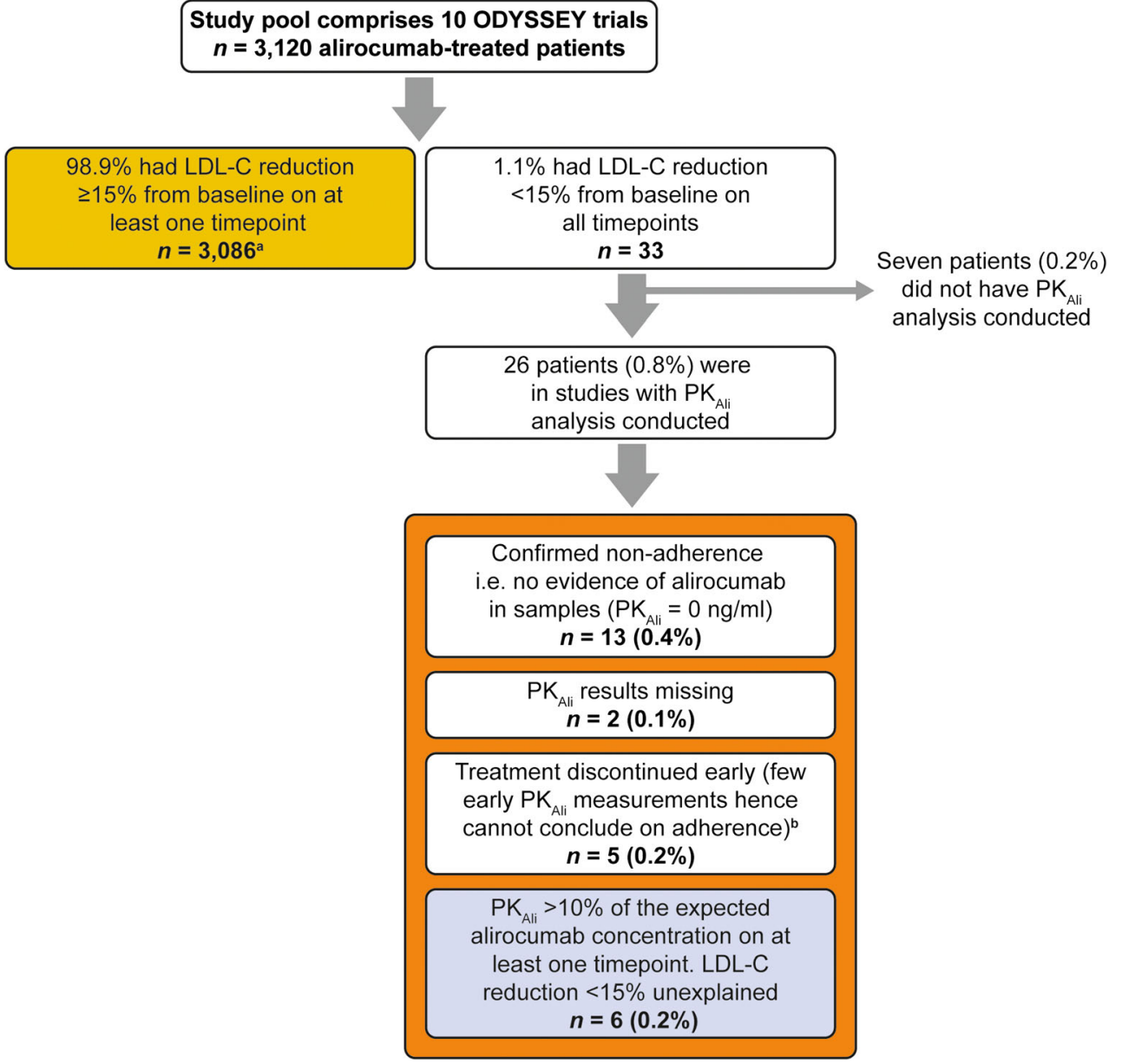

\section{Discussion}

Among the 3120 patients evaluated, $98.9 \%$ had $\geq 15 \%$ LDL-C lowering (responsiveness) to alirocumab. Of the 33 patients with apparent hyporesponsiveness, 27 had undetectable or missing alirocumab levels, absence of pharmacokinetics analyses, or early treatment discontinuation. Whether these patients had received alirocumab could not be concluded, either because no pharmacokinetics analysis was performed or there were too few $\mathrm{PK}_{\mathrm{Ali}}$ values.

\section{Limitations}

This post-hoc analysis, as well as the studies included in the analysis, was not designed to assess adherence to concurrent LLTs such as statins or ezetimibe. Alirocumab pharmacokinetics data were not planned per protocol in all studies.

\section{Clinical Implications}

Non-responsiveness to fully human PCSK9 monoclonal antibodies is rare. When non-responsiveness to PCSK9 monoclonal antibodies does occur, a worry among clinicians is the possible presence of anti-drug antibodies, especially given that PCSK9 monoclonal antibodies are biologics with antigenic potential. A prior report by Shapiro et al. [19] evaluated potential causes of hyporesponsiveness among 17 adults with cardiovascular disease $(n=14)$ and/or familial hypercholesterolemia $(n=9)$ treated with a PCSK9 inhibitor (12 patients received alirocumab and 5 patients received evolocumab). The authors concluded that because total PCSK9 levels typically rise with inhibition of PCSK9 via monoclonal antibodies (due to the antibody binding to PCSK9 in the circulation), this may assist in diagnosing potential causes of hyporesponsiveness. Such an approach may be advantageous in that PCSK9 levels are commercially available through specialty laboratories accessible to clinicians. Conversely, PCSK9 monoclonal antibody levels, and levels of anti-drug antibodies to PCSK9 monoclonal antibodies, are typically available only within the research setting. Furthermore, the presence of anti-drug antibodies does not necessarily mean the anti-drug antibody diminishes the effectiveness of the PCSK9 monoclonal antibody treatment. In fact, because "neutralizing" anti-drug antibodies are often defined by how antibody binding takes place, and not defined by their clinical effects, then even the presence of "neutralizing" anti-drug antibodies to PCSK9 inhibitor monoclonal antibodies may not necessarily account for diminished 


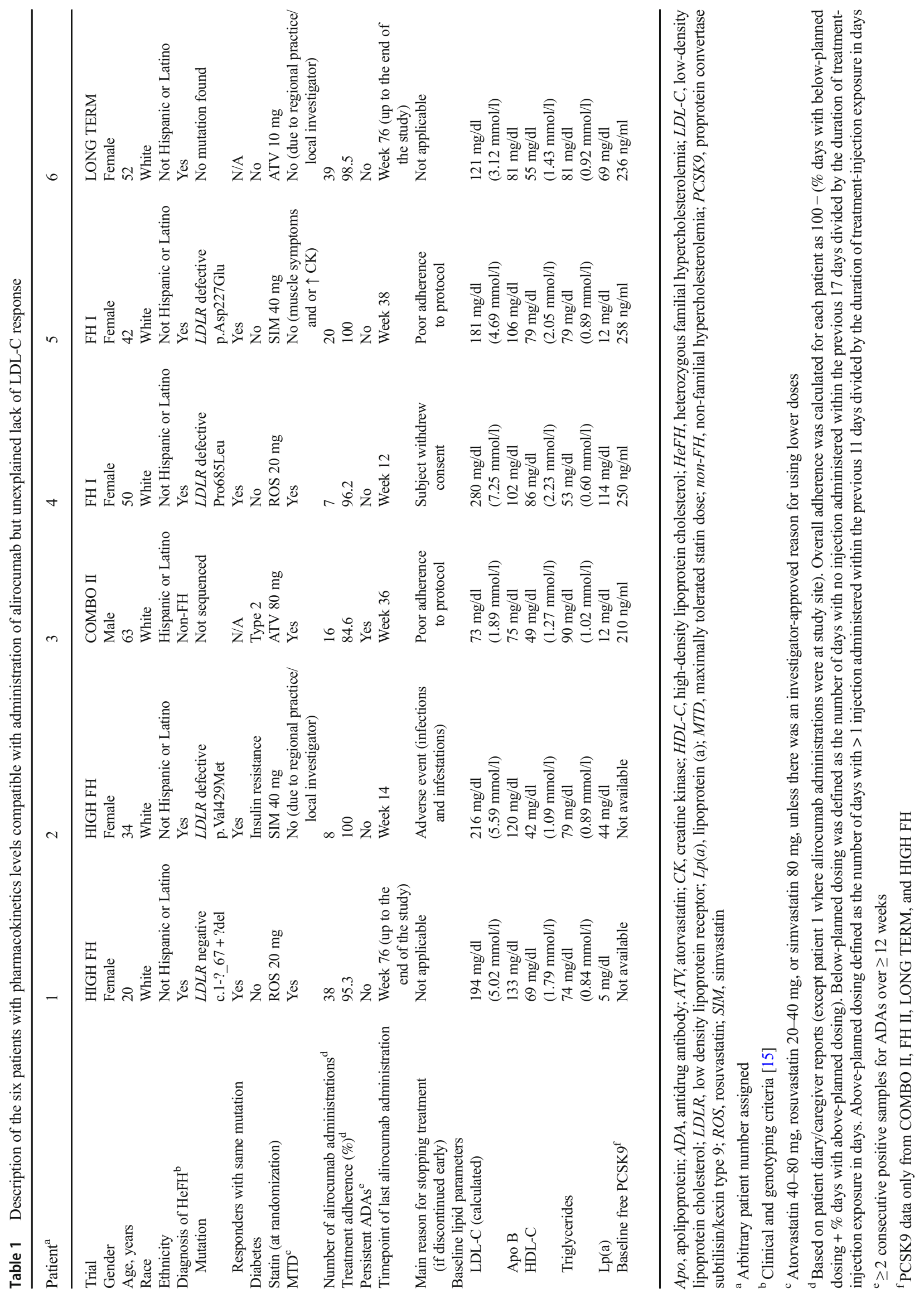


lipid-lowering effects. Finally, this current report suggests that in the event anti-drug antibodies to PCSK9 inhibitors do occur, their presence is, at best, a very rare potential cause of hyporesponsiveness. Other potential causes are far more likely.

All things considered, how might clinicians best evaluate a patient with apparent hyporesponsiveness to a PCSK9 monoclonal antibody? Based upon this data, one practical approach may be for the patient and medical staff to begin with a 4-week off PCSK9 inhibitor "stabilization" period, wherein other potential lipid-altering confounders are stabilized, including optimal management of diabetes mellitus, adherence to thyroid replacement therapy, and no significant change in nutrition or physical activity. During this 4-week period while off PCSK9 monoclonal antibody therapy, the medical staff should ensure an accurate 4-week pill count of concurrently administered statin and/or other lipid-altering drugs. This can be facilitated by patient self-records of pill intake, family-witnessed/documented lipid-altering drug intake, pill bottle counts by the medical staff, and pharmacy reconciliation of lipid-altering drugs and doses. Also during this 4-week stabilization, the medical staff should directly obtain, and properly store, the prescription PCSK9 monoclonal antibody. Once it is ensured that the patient was stabilized for 4 weeks, that the patient has adhered to concurrent lipid-altering drug therapies for 4 weeks, and that functional PCSK9 monoclonal antibody is ready for use, then a baseline lipid profile blood level [including LDL-C and lipoprotein(a)] should be obtained directly by the medical staff (not an outside laboratory). The patient should then receive PCSK9 monoclonal antibody administered by another highly trained medical staff member-verified and witnessed by an independent highly trained medical staff member. The same lipid profile should then be repeated at 2 and 4 weeks afterwards, while maintaining optimal management of secondary causes of dyslipidemia, maintaining no significant change in nutrition or physical activity, and a strict pill count to absolutely ensure the patient is maintaining concurrently administered statin and/or other lipid-altering drugs. Based upon the current report above, such a practical approach is likely to resolve the vast majority of cases of apparent hyporesponsiveness to a PCSK9 monoclonal antibody.

If, despite the above approach, the reduction in LDL-C levels remains $<15 \%$, then based upon the findings of Shapiro et al., PCSK9 levels could be obtained within 1-5 days after administration of PCSK9 monoclonal antibody therapy, and repeated at least 2 weeks off PCSK 9 monoclonal antibody therapy. If the plasma PCSK9 on:off ratio is $<2$ (or PCSK $9<1200 \mathrm{ng} / \mathrm{ml}$ ), then this suggests a lack of systemic exposure to the PCSK9 monoclonal antibody, as might occur from inactive drug, poor training of and poor injection technique by the patient and medical staff, dermatologic pathology impairing systemic absorption, problems with drug target recognition, or possible anti-drug antibodies. Conversely, if the plasma PCSK9 on:off ratio is $>2$ or PCSK9 $>1200 \mathrm{ng} / \mathrm{ml}$, then this suggests the patient has received the intended PCSK9 monoclonal antibody therapy. Potential causes of LDL-C hyporesponsiveness to functional PCSK9 monoclonal antibody therapy in patients with systemic exposure include potential exaggerated PCSK9 secretion, or mutations/ dysfunction of PCSK9, LDL receptor, apolipoprotein B, and/or apolipoprotein E.

\section{Conclusion}

In this analysis, treatment hyporesponsiveness in lowering LDL-C could be attributed to lack of receipt of alirocumab, possible lack of adherence to concurrent LLTs, a theoretical, rare possibility of biological non-responsiveness due to persistent antidrug antibodies, or other causes, as yet unidentified.

Acknowledgements The authors would like to thank the patients, their families, and all investigators involved in this study. The following people from the study sponsors provided editorial comments on the manuscript: Michael Howard (Sanofi), L. Veronica Lee (Sanofi), Corinne Hanotin (Sanofi), Robert Pordy (Regeneron Pharmaceuticals, Inc.), and Carol Hudson (Regeneron Pharmaceuticals, Inc.). Medical writing assistance and editorial support, under the direction of the authors, were provided by Aparna Shetty, PhD, of Prime (Knutsford, UK), funded by Sanofi and Regeneron Pharmaceuticals, Inc., according to Good Publication Practice guidelines (http://annals.org/aim/article/2424869/good-publicationpractice-communicating-company-sponsored-medical-research-gpp3). Sanofi and Regeneron Pharmaceuticals, Inc. were involved in the study design, collection, analysis, and interpretation of data, as well as data checking of information provided in the manuscript. The authors, who had unrestricted access to study data, were responsible for all content and editorial decisions and received no honoraria related to the development of this publication. Data from this analysis were previously presented as a poster at the 2017 American Heart Association Scientific Sessions, November 11-15, Anaheim, California.

Author Contributions Harold E. Bays was involved in the concept, design, data acquisition and interpretation, and in drafting the manuscript. Robert S. Rosenson was involved in the concept, design, and interpretation of the data. Marie T. Baccara-Dinet and Michael J. Louie were involved in the interpretation of the data. Desmond Thompson analyzed the data. G. Kees Hovingh was involved in the concept, design, and data acquisition and interpretation. All authors provided critical review of drafts and approved the final version for submission.

Funding This analysis and the ODYSSEY studies were funded by Sanofi and Regeneron Pharmaceuticals, Inc.

\section{Compliance with Ethical Standards}

Conflict of Interest Harold E. Bays has served as a consultant and/or speaker to Alnylam, Amarin, Amgen, AstraZeneca, Eisai, Eli Lilly, Merck, Novartis, NovoNordisk, Regeneron Pharmaceuticals, Inc., Sanofi, and Takeda and has received research grants from Amarin, Amgen, Ardea, Arisaph, Catabasis, Cymabay, Eisai, Elcelyx, Eli Lilly, Esperion, Hanmi, Hisun, F. Hoffman LaRoche, Home Access, Janssen, Johnson and Johnson, Merck, Necktar, Novartis, Novo Nordisk, Omthera, Orexigen, Pfizer, Pronova, Regeneron Pharmaceuticals, Inc., Sanofi, Takeda, TIMI, VIVUS, and Wpu Pharmaceuticals. 
Robert S. Rosenson has received research grants from Amgen, AstraZeneca, Esperion, Medicines Company, Regeneron Pharmaceuticals, Inc., and Sanofi; has served as a consultant/on advisory boards for Amgen, Akcea, AstraZeneca, CVS Caremark, Easy Vitals, Eli Lilly, and Sanofi; and receives royalties from UpToDate.

Marie T. Baccara-Dinet is an employee and stockholder of Sanofi.

Michael J. Louie is an employee and stockholder of Regeneron Pharmaceuticals, Inc.

Desmond Thompson is a consultant to Medical Affairs at Regeneron Pharmaceuticals, Inc.

G. Kees Hovingh's institution has received payment for conducting clinical trials from Sanofi, Regeneron Pharmaceuticals, Inc., Amgen, Pfizer, Kowa, Genzyme, Ionis Pharmaceuticals, Roche, Eli Lilly, Aegerion, Synageva, and AstraZeneca and for lectures and/or advisory panel participation from Amgen, Sanofi, Pfizer, and Roche.

Open Access This article is distributed under the terms of the Creative Commons Attribution 4.0 International License (http://creativecommons. org/licenses/by/4.0/), which permits unrestricted use, distribution, and reproduction in any medium, provided you give appropriate credit to the original author(s) and the source, provide a link to the Creative Commons license, and indicate if changes were made.

\section{References}

1. Trompet S, Postmus I, Slagboom PE, Heijmans BT, Smit RA, Maier AB, et al. Non-response to (statin) therapy: the importance of distinguishing non-responders from non-adherers in pharmacogenetic studies. Eur J Clin Pharmacol. 2016;72:431-7.

2. Simon JA, Lin F, Hulley SB, Blanche PJ, Waters D, Shiboski S, et al. Phenotypic predictors of response to simvastatin therapy among African-Americans and Caucasians: the cholesterol and pharmacogenetics (CAP) study. Am J Cardiol. 2006;97:843-50.

3. Jones PH, Bays HE, Chaudhari U, Pordy R, Lorenzato C, Miller K, et al. Safety of Alirocumab (a PCSK9 monoclonal antibody) from 14 randomized trials. Am J Cardiol. 2016;118:1805-11.

4. Ray KK, Ginsberg HN, Davidson MH, Pordy R, Bessac L, Minini $\mathrm{P}$, et al. Reductions in Atherogenic lipids and major cardiovascular events: a pooled analysis of 10 ODYSSEY trials comparing Alirocumab with control. Circulation. 2016;134:1931-43.

5. Bays H, Gaudet D, Weiss R, Ruiz JL, Watts GF, Gouni-Berthold I, et al. Alirocumab as add-on to atorvastatin versus other lipid treatment strategies: ODYSSEY OPTIONS I randomized trial. J Clin Endocrinol Metab. 2015;100(8):3140-8.

6. Cannon CP, Cariou B, Blom D, McKenney JM, Lorenzato C, Pordy $\mathrm{R}$, et al. Efficacy and safety of alirocumab in high cardiovascular risk patients with inadequately controlled hypercholesterolaemia on maximally tolerated doses of statins: the ODYSSEY COMBO II randomized controlled trial. Eur Heart J. 2015;36:1186-94.

7. Farnier M, Jones P, Severance R, Averna M, Steinhagen-Thiessen E, Colhoun HM, et al. Efficacy and safety of adding alirocumab to rosuvastatin versus adding ezetimibe or doubling the rosuvastatin dose in high cardiovascular-risk patients: the ODYSSEY OPTIONS II randomized trial. Atherosclerosis. 2016;244:138-46.

8. Ginsberg HN, Rader DJ, Raal FJ, Guyton JR, Baccara-Dinet MT, Lorenzato C, et al. Efficacy and safety of alirocumab in patients with heterozygous familial hypercholesterolemia and LDL-C of $160 \mathrm{mg} / \mathrm{dl}$ or higher. Cardiovasc Drugs Ther. 2016;30:473-83.

9. Kastelein JJ, Ginsberg HN, Langslet G, Hovingh GK, Ceska R, Dufour R, et al. ODYSSEY FH I and FH II: 78 week results with alirocumab treatment in 735 patients with heterozygous familial hypercholesterolaemia. Eur Heart J. 2015;36:2996-3003.

10. Kereiakes DJ, Robinson JG, Cannon CP, Lorenzato C, Pordy R, Chaudhari U, et al. Efficacy and safety of the proprotein convertase subtilisin/kexin type 9 inhibitor alirocumab among high cardiovascular risk patients on maximally tolerated statin therapy: the ODYSSEY COMBO I study. Am Heart J. 2015;169:906-15. e13

11. Moriarty PM, Thompson PD, Cannon CP, Guyton JR, Bergeron J, Zieve FJ, et al. Efficacy and safety of alirocumab vs ezetimibe in statin-intolerant patients, with a statin rechallenge arm: the ODYSSEY ALTERNATIVE randomized trial. J Clin Lipidol. 2015;9:758-69.

12. Robinson JG, Farnier M, Krempf M, Bergeron J, Luc G, Averna M, et al. Efficacy and safety of alirocumab in reducing lipids and cardiovascular events. N Engl J Med. 2015;372:1489-99.

13. Roth EM, Taskinen MR, Ginsberg HN, Kastelein JJ, Colhoun HM, Robinson JG, et al. Monotherapy with the PCSK9 inhibitor alirocumab versus ezetimibe in patients with hypercholesterolemia: results of a 24 week, double-blind, randomized phase 3 trial. Int J Cardiol. 2014;176:55-61.

14. FDA. Guidelines for the clinical evaluation of lipid-altering agents in adults and children: center for drug evaluation and research. 1990.

15. Defesche JC, Stefanutti C, Langslet G, Hopkins PN, Seiz W, Baccara-Dinet MT, et al. Efficacy of alirocumab in 1191 patients with a wide spectrum of mutations in genes causative for familial hypercholesterolemia. J Clin Lipidol. 2017;11:1138-346.

16. Raal F, Scott R, Somaratne R, Bridges I, Li G, Wasserman $\mathrm{SM}$, et al. Low-density lipoprotein cholesterol-lowering effects of AMG 145, a monoclonal antibody to proprotein convertase subtilisin/kexin type 9 serine protease in patients with heterozygous familial hypercholesterolemia: the reduction of LDL-C with PCSK9 inhibition in heterozygous familial hypercholesterolemia disorder (RUTHERFORD) randomized trial. Circulation. 2012;126:2408-17.

17. Rey J, Poitiers F, Paehler T, Brunet A, DiCioccio AT, Cannon CP, et al. Relationship between low-density lipoprotein cholesterol, free proprotein convertase subtilisin/kexin type 9 , and alirocumab levels after different lipid-lowering strategies. J Am Heart Assoc. 2016;5: e003323.

18. Roth EM, Goldberg AC, Catapano AL, Torri A, Yancopoulos GD, Stahl N, et al. Antidrug antibodies in patients treated with alirocumab. N Engl J Med. 2017;376:1589-90.

19. Shapiro MD, Miles J, Tavori H, Fazio S. Diagnosing resistance to a proprotein convertase subtilisin/kexin type 9 inhibitor. Ann Intern Med. 2018;168:376-79. 